\title{
Hereditary Ovarian Cancers
}

\author{
T.RAJKUMAR,
}

Ovarian cancer is the third most common cancer seen among the women in Madras, (Madras Metropolitan Tumour Registry), Delhi and Mumbai, with an AAR (age adjusted rate) of 5.3 / 100,000 in Madras (Shanta and Swaminathan, 2005). It is also the number one killer among the gynecological cancers. Hereditary ovarian cancers account for less than $10 \%$ of the ovarian cancers. Hereditary ovarian cancers can arise due to the mutations in the following genes: BRCA1, BRCA2 (Hereditary breast and ovarian cancers - epithelial cancer), MSH2, MLH1, MSH6, PMS1, PMS2 (Hereditary Non-polyposis colon cancer, Lynch syndrome 2 - epithelial cancer), STK11 (Peutz Jeghers syndrome - sex cord tumours and Sertoli-Leydig cell tumours ), p53 (Li Fraumeni syndrome - germ cell tumours of the ovary), AT (Ataxia Telangiectasia epithelial, stromal, germ cell), PRKAR1A (Carney complex 1 - epithelial) and PTCH
(Gorlin's syndrome - fibromas, rarely fibrosarcomas).

Mutation analysis for BRCA1, BRCA2, MSH2, MLH1 can help identify the high risk families and we can then offer predictive testing for the unaffected members of the family, to see if they carry the same deleterious mutation seen in their affected family member. If they also carry the same deleterious mutations, risk reduction strategies will need to be undertaken for the mutation carriers.

Currently the management of hereditary epithelial ovarian cancers is no different from that of the sporadic cancers. Some studies have suggested a better response to platinum based therapy if they carried BRCA1 mutations. On the contrary, mutations in MSH2 and MLH1 may carry a poorer response to platinum based therapy. 